Une cartographie des indéfinis free choice du français
Claude Muller (Bordeaux \& CNRS: UMR CLLE, 5263)

( Syntaxe et Sémantique, 10, 2009, p. 65-78, Presses Universitaires de Caen).

abstract.

We describe the principal properties of the indefinite series used in the free-choice constructions in French. It requires some modifications in the cartography of French indefinite pronouns proposed in Haspelmath 1997. The two principal properties, non-individuation and widening, are equally observed for the two major series. A detailed account of their functions shows that n'importe has prototypic FC properties, when the major other series, que ce soit, has more restricted condtions of use.

résumé.

On examine les principales propriétés des indéfinis des constructions "de libre choix" en français. Cela implique de modifier la cartographie des indéfinis du français proposée dans Haspelmath 1997. Les deux principales propriétés, non-individuation et élargissement, se retrouvent dans les deux principales séries. Une analyse plus détaillée montre que la série n'importe a des propriétés bien distinctes et prototypiques, alors que la série que ce soit est beaucoup plus contrainte dans ces contextes.

1. La représentation des indéfinis du français dans Haspelmath 1997.

Dans une perspective typologique basée sur la comparaison des formes et des collocations des pronoms indéfinis de 140 langues, Haspelmath a proposé une cartographie basée sur un schéma implicationnel général des principaux emplois fonctionnels des indéfinis. A une extrémité du schéma figurent les emplois de type free-choice (ou pronoms "de libre choix"). Les principales séries d'indéfinis sont représentés par des zones en intersection puisque dans la plupart des fonctions, plusieurs formes indéfinies sont possibles. Le principe essentiel selon lequel cette cartographie générale a pu être établie est l'obligation d'une représentation sans discontinuité des diverses séries indéfinies dans les langues du monde, mettant ainsi en évidence, par proximité, les relations de contiguïté des emplois fonctionnels.

Le schéma se limite pour le français à la série des formes utilisées dans les emplois spécifiques aussi bien factif qu'irréels (quelqu'un, quelque chose, un $N$ ), celle qui est assez largement coïncidente avec la polarité négative (qui/quoi que ce soit), et la série des négatifs, représentée ici par personne, débordant largement sur d'autres domaines (il s'agit classiquement des emplois "à polarité négative", ou encore décrits comme "positifs", des indéfinis négatifs). A l'extrémité inférieure droite, on trouve aussi la série caractéristique des emplois free-choice -désormais FC- (n'importe qui/ quoi, n'importe quel...). Cette série est utilisable, d'après ce schéma, dans deux classes d'emplois, les emplois en comparative et les emplois FC. Dans les emplois FC, la série n'importe est en concurrence avec la série que ce soit.

On notera aussi que la notion de polarité négative a été rejetée comme trop imprécise dans ces schémas: la polarité négative est subdivisée et représente ici l'ensemble des domaines englobés par la distribution de personne: négation directe, négation indirecte, comparatifs, conditionnel, questions. 


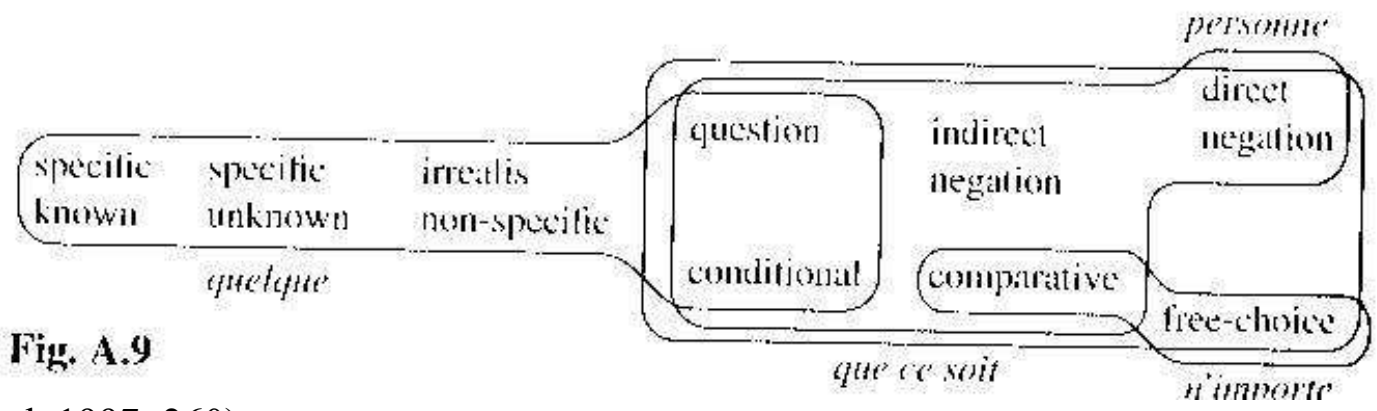

(Haspelmath 1997: 260)

2. Une représentation modifiée des séries à emplois free-choice.

Les emplois free-choice sont des constructions d'indéfinis de tout domaine qui ont comme les indéfinis à polarité négative des propriétés de non spécification des objets, sans pour autant partager les autres propriétés typiques de la polarité négative, comme on le verra ci-dessous.

La série la plus représentative en français de ces indéfinis est la série n'importe.

La carte d'Haspelmath comporte un certain nombre d'inexactitudes sur la distribution des indéfinis du français. Entre autres, la série de type quelqu'un est également utilisable en contexte de négation indirecte, et la série des négatifs n'a probablement autant d'extension, du moins en français moderne, que celle figurée ici, couvrant l'ensemble des domaines à polarité: on peut en tout cas en exclure les contextes de type conditionnel: *Si personne venait,.... Pour ce qui nous intéresse ici, les emplois liés à la fonction free-choice, il faut au contraire étendre bien au-delà de ce qui est indiqué l'utilisation des indéfinis de type n'importe qui. La carte modifiée pour les seules deux séries qui nous intéressent serait la suivante:

\begin{tabular}{|c|c|c|}
\hline $\begin{array}{l}\text { specífic } \\
\text { known }\end{array}$ & $\begin{array}{l}\text { specifie } \\
\text { unknown }\end{array}$ & $\begin{array}{l}\text { irrealis } \\
\text { non-specific }\end{array}$ \\
\hline $\begin{array}{l}\text { quelqu's } \\
\text { quelque }\end{array}$ & & \\
\hline
\end{tabular}

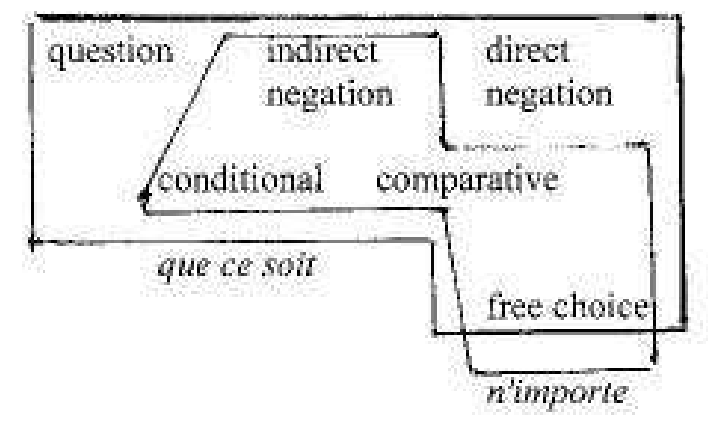

Ainsi, l'utilisation possible de la série n'importe (sans mise en évidence de son sens discriminant, avec l'équivalence approximative des indéfinis de type qui que ce soit) couvre les constructions de type conditionnel (dans le sens ou n'importe alterne sans changement significatif de sens avec quoi que ce soit en (1)):

1) Si n'importe quoi vous gêne, dites-le nous.

La distribution s'étend aussi aux constructions à négation "indirecte" dans leurs deux formes: négation super-ordonnée ou mot négatif sémantique:

2) Je ne crois pas que n'importe qui soit venu. (=pour moi, personne n'est venu)

3) Il est incapable de faire du mal à n'importe qui (= à quiconque, à qui que ce soit). 
Par contre, dans les emplois de type "négation directe", c'est le sens spécifique, discriminant, qui apparait de préférence ${ }^{1}$ :

4) On n'ouvre pas à n'importe qui (E. Vlachou, p. 148)

(on ouvre à certains, qu'on choisit, mais pas à n'importe qui)

Dans les questions, la série n'importe ne remplace pas de façon naturelle la série la plus appropriée dans les emplois à polarité, comme le montre (5):

5) Pourquoi serions-nous émus de quoi que ce soit ?

(Claudel, Tête d'or, 1901, Frantext)

Mettre n'importe quoi à la place de quoi que ce soit risque d'être mal interprété (difficilement au sens général et coextensif à tout ce qui est envisageable).

Les deux séries ont donc des distributions qui ne coïncident pas, même si elles partagent une propriété commune basique: la non-individuation dans un ensemble: le pronom ou déterminant désigne un individu interchangeable sur l'ensemble de référence.

\section{Les emplois free-choice.}

L'étiquette free-choice est utilisée par Kadmon \& Landman 1993 pour désigner les emplois de any qui n'entrent pas dans les domaines et les interprétations à polarité négative. L'étiquette a déjà été utilisée auparavant avec ce sens pour le même item par Carlson $1981^{2}$. Elle désigne une gamme d'emplois dont certains sont de type générique (c'est le seul emploi discuté par Kadmon \& Landman 1993):

6) Any owl hunts mice

Toute chouette chasse des souris

L'indéfini signale l'appropriation du prédicat pour tout élément de l'ensemble de référence.

Le français utilise tout dans ce cas. On peut dans les mêmes contextes utiliser n'importe, peutêtre aussi la série moins usuelle quelque...que ce soit (plus aisément ${ }^{3}$ quand elle est circonscrite à un sous-ensemble délimité par une expansion comme en (9))

7) Quelque fraudeur que ce soit sera puni.

8) N'importe quel fraudeur sera puni. examinée.

9) Quelque demande que ce soit ayant été déposée dans les délais requis a bien été

Dans d'autres cas, l'emploi n'est pas générique: il y a dissociation entre le choix et la réalisation, ou entre un "avant" dans un monde virtuel, et un "après" dans le monde réel. Par exemple, dans (10):

10) Prenez n'importe quelle carte.

\footnotetext{
${ }^{1}$ E. Vlachou traduit par (4) anybody : c'est certainement exclu ici, sauf rupture de construction et emphase, mettant n'importe qui hors de portée de la négation. On peut quand même dire: je n'ai pas vu n'importe lequel d'entre eux, au sens: absolument aucun d'entre eux, avec une interprétation focalisée et une intonation marquée sur la finale: c'est que l'indéfini est alors hors de portée de la négation: n'importe lequel, je ne l'ai pas vu. La négation ne porte donc pas sur la prédication de sens discriminant.

${ }^{2}$ Sur les emplois FC en général, cf. la monographie d'E. Vlachou 2007; autre référence: Jayez \& Tovena 2005. Sur le français, une étude pionnière (sans employer l'étiquette FC): Paillard 1997; Muller 2006, 2007a.

${ }^{3}$ Si la phrase n'est pas générique, il faut une extension possible à tout élément virtuel (cf. Kleiber \& Martin 1977).
} 
Le choix est ouvert à toute carte, mais il est normalement compris qu'une seule carte est à prendre ${ }^{4}$. La non-individuation ne vaut que pour le choix, la quantité étant déterminée.

Dans d'autres contextes, la quantité dans le monde réel est limitée pragmatiquement aux possibles cas de réalisation projetée, comme en (11), ou à une énumération ouverte sur une suite indéfinie (12):

11) Je défie qui que ce soit de me contredire.

12) Ils regardaient la bière ou leur canne, ou n'importe quoi (Camus, L'Etranger, 1942, Frantext).

4. Propriétés des emplois free-choice: non individuation et élargissement.

La propriété la plus générale est, au moins dans un "espace mental", l'extension indéterminée de la référence à partir d'un objet singulier, décrit comme interchangeable. Cette propriété: la non-spécification, ou encore selon la terminologie de Kadmon \& Landman, la nonindividuation, se retrouve non seulement dans les emplois free-choice, mais aussi dans les emplois des indéfinis dans la polarité négative. Ce qui singularise les emplois FC, c'est que la polarité n'est pas négative.

Dans les conditions normales d'énonciation, la non-individuation produit un effet d'élargissement: une extension de la référence à partir d'un objet variable, sur tout l'ensemble. C'est ce qui se passe dans les emplois génériques. Dans ces emplois, on trouve indifféremment tout, n'importe, quelque...que ce soit. Mais il peut y avoir des limitations selon les séries. Ainsi, la série quelque...que ce soit est inadaptée aux ensembles à extension limitée, ce qui la distingue de la série n'importe:

13) N'importe quel imbécile musclé et dur au mal s'en tirait mieux que lui. (R. Guérin, L'Apprenti, 1946, Frantext)

14) ?*Quelque imbécile que ce soit s'en tirait mieux que lui.

14) N'importe quelle fille est plus astucieuse que les garçons dans cette classe.

15) N'importe quelle demande a bien été examinée.

On notera ainsi que (15) ne requiert pas, contrairement à (9), une expansion caractérisant plus étroitement le domaine de référence.

Pour les autres séries, il y a également des particularités. Par exemple, avec un quelconque, on ne trouve pas d'élargissement à l'ensemble dans les contextes sans renversement des échelles (à polarité positive) comme les emplois génériques:

16) Un quelconque fraudeur sera puni.

On utilisera (16) pour désigner un individu interchangeable, mais pas tout individu, par exemple dans le contexte suivant: Le gouvernement se doit de réprimer la fraude, mais il se contentera de faire un exemple: un quelconque fraudeur sera puni et la répression s'arrêtera là.

Sinon, en contexte à polarité, le sens de un quelconque se confond avec celui de quelque que ce soit.

17) Je doute que cela présente un quelconque intérêt.

Il en va de même avec quelque au singulier, qui offre de multiples contraintes et se caractérise par une sorte de "non-individuation" (dans les termes de Kadmon \& Landman) qui reste

\footnotetext{
${ }^{4}$ Cela ressemble aux emplois non spécifiques des indéfinis, mais avec une importante différence: les non spécifiques ne spécifient pas sans pourtant poser que toute unité équivaut aux autres. Par exemple: Georges rêve d'épouser une tahitienne

(au sens non spécifique: il n'en connaît aucune). On ne peut dire cependant avec le même sens: Georges rêve d'épouser n'importe quelle tahitienne.
} 
locale, sans élargissement à l'ensemble (sinon, ce serait quelque que ce soit). Sur ce terme, voir Culioli 1983, Corblin 2004, Muller 2007b.

18) Vous croyez que tout cela offre quelque intérêt? (Pontalis, L'Enfant des limbes, 1998, Frantext)

\section{L'élargissement dissocié selon les espaces.}

Une des particularités des emplois FC non génériques est la dissociation entre l'espace ouvert au choix et celui de la réalisation. Dans l'exemple suivant, il y a élargissement dans l'espace du choix, pas dans la réalisation future, qui obéit aux contraintes pragmatiques de quantification dans le monde réel (il s'agit de préparer un ou deux plats):

19) Fais n'importe quoi à déjeuner (Colette, Chambre d'hôtel, 1940, Frantext).

L'impératif est un contexte qui permet cette dissociation. Avec cette interprétation qui dissocie l'espace mental du choix de la quantité dans le réel, on ne peut pas utiliser les formes tout et la série que ce soit, parce que ces formes doivent coordonner les quantifications virtuelle et réelle:

$19 ')$ *Fais quoi que ce soit à déjeuner.

Par contre, si l'espace du choix est prolongé par un espace du réel qui peut référer à chaque occurrence indéfinie de l'espace du choix, la série que ce soit est acceptable:

20) C'est faisable, demandez quoi que ce soit, ça sera fait (Google)

21) Vous me demandez quoi que ce soit sur quelqu'un de l'immeuble, je sais.

(Libération, 23/10/06)

Les constructions (20), (21) sont des conditionnelles cachées: l'assertion finale réfère chaque fois à une occurrence particulière de la variable représentée par l'indéfini: si vous demandez cela, ça sera fait. Ainsi, la quantification du réel est corrélée à la liberté de choix de la variable. Cela vaut évidemment pour les vraies conditionnelles:

22) Lorsque vous demandez quoi que ce soit, veillez à utiliser des termes tels que 'could you', et 'please'. (Google)

Les contextes factifs d'actions répétées déclenchant un événement quelconque font également correspondre une quantification de type FC à son aboutissement coréférent. Cependant, on ne peut librement utiliser les deux séries dans ces contextes factifs. Alors que n'importe, la série prototypique des emplois FC, est toujours possible, la série que ce soit est parfois exclue. Le contraste entre (23) et (24) le montre:

23) Qu'on fasse allusion à quoi que ce soit au sujet de son père le met de mauvaise humeur.(M. Charef, Le Thé au harem d'Archi Ahmed1983, Frantext)

24) En effet, toutes les cinq chansons, l'animateur appelle n'importe qui, au hasard du Bottin, afin qu'on lui cite le montant exact de la somme en jeu...(Ernaux, Journal du dehors, 1993, Frantext)

On peut employer n'importe quoi en (23). Par contre, on ne peut pas remplacer n'importe qui en (24) par qui que ce soit. La différence entre (23) et (24) tient peut-être au caractère non borné pragmatiquement de la quantification attachée à quoi que ce soit, qui est une extrémité scalaire (la moindre chose conviendrait aussi): la quantification est de type universel, extensible virtuellement à toute situation semblable. En (24), on a typiquement une quantification bornée, régulée et sans extension hors du réel. Dans ce type d'emploi, typiquement FC, la série que ce soit n'est pas utilisable.

\section{L'orientation scalaire.}


Le lien entre la scalarité et les emplois FC n'est pas absolument évident. Haspelmath (1997:117), suivant en cela Fauconnier, à propos de l'analyse par Fauconnier 1976 de any, justifie la proximité des emplois $\mathrm{FC}$ et des emplois à polarité par une propriété commune, et une différence d'orientation. La polarité et FC correspondent tous deux à des extrémités d'échelles. Cependant, alors que les contextes à polarité négative ont des échelles orientées vers zéro, les échelles FC sont du type de la polarité positive.

On peut le montrer sur un type de construction qui permet une interprétation soit FC soit à polarité, aussi bien en français qu'en anglais: les conditionnelles. Haspelmath reprend l'exemple de Fauconnier (1975: 373) qui a deux lectures avec le même indéfini any:

$25)$ If she can solve any problem, she'll get a prize

Dans l'interprétation à polarité, any peut se gloser par le moindre problème et revient à une quantification existentielle; dans l'interprétation FC, any correspondrait à l'extrémité opposée de l'échelle, représentable par le problème le plus difficile, et signifie alors la quantification universelle: tout problème. La solution unitariste, et élégante, proposée par Fauconnier pour expliquer l'ambivalence de any, est donc que ce terme signifie toujours une extrémité d'échelle quelconque ${ }^{5}$. Selon les propriétés contextuelles, la quantification sera alors existentielle ou universelle. La quantification existentielle de ces constructions est liée à une échelle quantitative, et non qualitative.

On peut faire la même analyse pour le français, aussi bien avec la série que ce soit qu'avec la série n'importe. Dans les exemples (26) et (27), on a dans la conditionnelle la même ambiguïté, avec une interprétation sélectionnée par la principale:

26) Si quoi que ce soit vous irrite, vous feriez mieux de rester chez vous.

26') Si n'importe quoi vous irrite, vous feriez mieux de rester chez vous.

27) Si quoi que ce soit vous irrite, prévenez-nous afin que nous puissions y remédier.

27') Si n'importe quoi vous irrite, prévenez-nous afin que nous puissions y remédier.

On constate que (26) et (26') ont une interprétation universelle, alors que celle de (27), (27') est existentielle. Dans les deux cas, on a affaire à une extrémité d'échelle: d'ailleurs, le superlatif la moindre chose serait approprié dans les deux cas. La différence essentielle est dans l'application à la quantification: en (26), la moindre chose vaut pour toute l'échelle, alors qu'en (27) on aura une interprétation existentielle: s'il y a une chose qui vous irrite. Cette interprétation est conforme aux analyses de Fauconnier (1976: 42) supposant une échelle existentielle de quantité orientée vers zéro dans les contextes à polarité. Les conditionnelles permettent l'une ou l'autre interprétation.

On remarque cependant que la scalarité ne différencie pas les deux séries, alors que la série n'importe est typiquement $\mathrm{FC}$, et la série que ce soit adaptée à la polarité négative.

Il me semble qu'il y a une différence entre les indéfinis relativement à la scalarité, précisément: la série n'importe y est indifférente, même si elle s'y plie dans les contextes induisant la polarité négative. Par contre, la série que ce soit fonctionne différemment: soit elle signifie une extrémité scalaire sans renversement, donc la quantification universelle, soit elle a des interprétations existentielles, mais alors exclusivement à scalarité orientée vers zéro.

Le premier point: l'absence de scalarité, est visible sur les constructions à élargissement dissocié. Dans les emplois comme (10), il n'y a aucune inférence de qualité ou de quantité:

(10) Prenez n'importe quelle carte

\footnotetext{
${ }^{5}$ Sur les nombreuses analyses de any, voir aussi Horn 2000, 2001, ainsi que Kadmon \& Landman 1993. Fauconnier se classe parmi les "unitaristes" comme Kadmon \& Landman. Beaucoup d'analyses traditionnelles en logique posent deux termes distincts, un any universel, et un any existentiel.
} 
(10) ne signifie pas:...même la plus importante, ou encore la moindre carte. L'interprétation pragmatique (une seule carte) est purement contextuelle et n'apparaît pas dans d'autres cas de FC à dissociation:

(10') Prenez n'importe quel outil, mais n'oubliez pas de le(s) remettre en place.

En (10'), la quantification est FC dans le choix, indéfinie dans le monde réel, sans scalarité. Dans ces emplois, que ce soit n'apparait pas: on lui préfère la série n'importe.

On trouve donc quelquefois la série que ce soit avec une interprétation indéfinie, y compris dans le monde réel, mais lorsque l'élargissement est possible dans des contextes à renversement scalaire. C'est ainsi que l'on peut comprendre l'exemple suivant de E.Vlachou:

28) Je suis même étonné que quoi que ce soit fonctionne.

(E. Vlachou, 2007: 194)

Le sens est bien ici: la moindre chose, avec une orientation vers zéro. Il s'agit d'un emploi à polarité classique.

Dans d'autres contextes, moins nettement interprétables comme des contextes à polarité négative, on retrouve néanmoins cette orientation scalaire vers zéro:

29) (votre argent) Il vaut mieux le mettre dans une banque sérieuse, lui faire produire quoi que ce soit. (T. Bernard, Monsieur Codomat, 1907, Frantext)

Le sens induit par quoi que ce soit est existentiel et l'emploi de cet indéfini induit une lecture à renversement d'échelle: si peu que ce soit, plutôt qu'une lecture neutre: un peu d'argent.

Au total donc: lorsqu'il n'y a pas scalarité, notamment dans les constructions à dissociation, on emploie exclusivement la série n'importe. La série que ce soit peut figurer avec un sens de quantification existentielle indéfinie dès lors que la polarité est négative.

\section{Les emplois qualificatifs.}

On doit distinguer entre les emplois à dissociation, où une lecture $\mathrm{FC}$ virtuelle précède une application spécifiée projetée dans le réel, et des constructions où les indéfinis sont en fait des indéfinis spécifiants masqués par l'utilisation de déterminants FC: simplement, l'indéfini FC est utilisé comme une épithète qualificative pour signifier que la qualité de l'indéfini n'a aucune importance.

Par exemple:

30) J'ai répondu n'importe quoi.

31) Il a sans doute mangé n'importe quoi qui l'a rendu malade.

Il s'agit d'indéfinis spécifiés dans lesquels le déterminant est qualificatif: J'ai répondu quelque chose, qui était n'importe quoi (cela n'avait aucune importance ou aucune pertinence.)

L'indéfini est existentiel et spécifié: en (31), on peut référer à ce qu'il a mangé. En (32) cidessous, on trouve côte à côte l'interprétation FC à élargissement universel (sur un ensemble pragmatiquement limité), suivi dans la seconde phrase de cet emploi qualificatif: dans la seconde phrase, factitive, quelqu'un a gagné. L'indéfini est qualifiant. Pour Vlachou, qui explicite ce sens par "a poor guy" - "un pauvre type", il y a une interprétation dépréciative, ce qui ne me semble pas le cas ici. Le sens de la phrase hors contexte est plutôt celle d'un "Monsieur Tout-le-monde" sans rien d'exceptionnel.

32) N'importe qui pouvait gagner. N'importe qui a gagné. (E. Vlachou 2007: 198)

On retrouve naturellement cet emploi dans des phrases habituelles:

33) Il raconte sa vie à n'importe qui.

Dans ces emplois, qui supposent une absence totale de discrimination qualitative, l'indéfini FC prend facilement des colorations péjoratives, correspondant aux marges qualitatives de l'ensemble de référence. C'est net en (34):

34) Vous travaillez comme une machine, et vous faites n'importe quoi. (Dhôtel, Le Village pathétique, 1943, Frantext) 
Le sens est nettement dépréciatif ici: ce que vous faites n'est pas bien fait.

Comment se différencient les deux séries d'indéfinis à emploi FC dans ces constructions? Il s'agit en fait, dans les constructions sans renversement d'échelle, pratiquement toujours de la série n'importe. La concurrence entre les deux séries apparaît dans les contextes aux marges de la polarité, comme dans (35) ci-dessous:

35) Comme si elle flirtait avec qui que ce soit! (Thérame, Bastienne, 1985, Frantext)

Dans cette phrase, on a les ingrédients habituels de la série que ce soit: élargissement à l'ensemble virtuel des personnes dans un contexte à polarité, avec une interprétation existentielle quantitative: elle ne flirte avec personne. Si on remplace l'indéfini par n'importe $q u i$, on a en plus de la lecture identique (quantification existentielle orientée vers zéro) une lecture sur une échelle de qualité:

35') Comme si elle flirtait avec n'importe qui!

La lecture qualificative devient donc accessible: elle flirte (peut-être) avec quelqu'un mais ce n'est certainement pas n'importe qui.

Il peut arriver, mais on est aux marges de l'accessibilité, que la série que ce soit soit utilisée avec un sens qualificatif, exclusivement en contexte non factif ${ }^{6}$ :

36) Laissez-moi plonger sans appât. Je vous rapporterai de ma plongée quoi que ce soit qui l'atteste et qui la prouve. (Gide, Thésée, 1946, Frantext)

37) Celui-ci cherchait quoi que ce soit de cinglant. (Gide, Les Faux-monneyeurs, 1925, Frantext)

38) Qu'est-ce qui me prend ce matin? Cette brusque envie d'écrire quoi que ce soit dans ce carnet...(Gide, Journal, II, 514)

Ces emplois ne correspondent pas à des contextes à polarité négative. Les indéfinis y sont de type existentiel irrealis. L'emploi reste marginal. On le retrouve même dans un format plus nettement qualificatif, avec un déterminant défini:

39) Il fallut des siècles pour produire ce quelque chose, pour dégager ce quoi que ce soit du chaos. (Gide, Journal, II, 1045)

\section{Conclusion.}

Les deux séries étiquetées à juste raison "free-choice" dans le tableau d'Haspelmath sont plus proches l'une de l'autre que son tableau ne l'indique: nous proposons une modification sur ce point, montrant que les deux séries sont concurrentes dans les domaines free-choice, mais aussi des comparatifs, des conditionnelles, et de la négation indirecte - donc partiellement sur ce qui relève de la polarité. Ces indéfinis partagent la propriété d'élargissement à l'ensemble de référence, mais la série que ce soit exige un ensemble ouvert sur des occurrences virtuelles alors que n'importe peut s'adapter à des ensembles limités pragmatiquement. En dehors des emplois génériques, on trouve des constructions free-choice à dissociation, avec libre choix dans l'espace virtuel, et une quantification spécifiée dans l'espace du réel. Ici aussi, c'est la série n'importe qu'on peut utiliser sans restrictions, alors que la série que ce soit suppose une applicabilité automatique de type condition / conséquence. L'orientation scalaire divise nettement les deux séries: la série que ce soit à quantification existentielle figure dans les contextes à renversement d'échelles, alors que la série n'importe s'adapte à toutes les situations, y compris les contextes sans scalarité. Enfin, les deux séries peuvent avoir des emplois qualificatifs, mais cela reste marginal avec la série que ce soit, alors que c'est courant avec la série n'importe.

Le tableau ci-dessous récapitule ces principales distinctions pour les indéfinis à possible emploi FC. Les + signalent des propriétés FC, et on constate que la série prototypique est bien

\footnotetext{
${ }^{6}$ Ce n'est peut-être pas un hasard si tous les exemples réels trouvés sont du même écrivain, André Gide.
} 
celle du type n'importe. 


\begin{tabular}{l|c|c|c|c|c} 
& tout & quelque & que ce soit & n'importe & $\begin{array}{c}\text { quiconque, } \\
\text { (un) quelconque }\end{array}$ \\
\hline $\begin{array}{l}\text { Elargissement } \\
\text { (polarité }+)\end{array}$ & + & - & + & + & + \\
\hline $\begin{array}{l}\text { Elargissement } \\
\text { (ensemble réel) }\end{array}$ & - & - & - & + & - \\
\hline $\begin{array}{l}\text { Dissociation } \\
(\exists x \text { dans un monde) }\end{array}$ & - & + & + & + & + \\
\hline $\begin{array}{l}\text { FC extensif au } \\
\text { domaine dissocié }\end{array}$ & & - & + & + & + \\
\hline $\begin{array}{l}\text { Quantification } \exists \text { neutr } \\
\text { (vs. orientée vers } 0)\end{array}$ & & + & - & + & + \\
\hline Emploi qualifiant & - & - & marginal & + & + \\
& & & & &
\end{tabular}

\section{Références.}

CARLSON G. (1981), "Distribution of Free-Choice Any" in papers from the Seventeenth Regional Meeting of the Chicago Linguistics Society, Hendrik, Masek \& Miller (eds.), CLS , 17, University of Chicago, p. 8-23.

CoRBLIN F. (2004), "Quelque". In Handbook of French Semantics, F. Corblin \& H. de Swart (eds.), Stanford, CSLI, p. 99-107.

Culioli A. (1983), "A propos de quelque". In Linguistique, énonciation: aspects et détermination, S. Fisher \& J.J. Franckel (éds.), Paris, EHESS, p. 21-29.

DAYAL V. (2004), “The Universal Force of Free-Choice Any, "Linguistic Variation Yearbook 4, 5-40.

HasPelmath M. (1997), Indefinite Pronouns, Oxford University Press, Oxford.

HORN L. R. (2000), "Pick a Theory (Not Just Any Theory)", in Negation and Polarity, L.R. Horn \& Y. Kato (eds), Oxford U.P., p. 147-192.

HORN L. R. (2001), "Any and (-)ever: Free choice and free relatives", IATL 15 (Proceeding of the 15th Annual Conference of the Israeli Association for Theoretical Linguistics), p. 71-111.

JAYEZ J. \& L. TOVENA (2005), "Free Choiceness and Non-Individuation", Linguistics and Philosophy, 28, p. 171.

KADMON N. \& F. LANDMAN (1993), "Any", Linguistics and Philosophy, 16, p. 353-422.

KLEIBER G. \& R. MARTIN (1977), "La quantification universelle en français", Semantikos, 2-1, p. 19-36.

LADUSAW W.A. (1980), Polarity Sensitivity as Inherent Scope Relations, New York, Garland.

MulLeR C. (2006), "Polarité négative et free choice dans les indéfinis de type que ce soit et n'importe", Langages, 162, p. 7-31.

MULLER C. (2007a), "Les indéfinis free choice confrontés aux explications scalaires, Travaux de linguistique, 54, p. $83-96$

MulLer C. (2007b), "Quelque, déterminant singulier", Cahiers de lexicologie, 90, p. 135-149.

PAILlARD D. (1997), "N'importe qui, n'importe quoi, n'importe quel N", langue Française, 116, p. 100-114.

TOVEna L., V. DÉPREZ \& J. JAYEZ (2004), "Polarity Sensitive Items", in: F. Corblin \& H. de Swart (Eds): Handbook of French Semantics, CSLI, Stanford, p. 391-416.

Vlachou E. (2007), Free Choice in and out of Context: Semantics and Distribution of French, Greek and English Free Choice Items, LOT, Utrecht. 